\title{
ESTABILIDAD A TRAVÉS DE AMBIENTES DE LAS PROPIEDADES REOLÓGICAS DE TRIGOS HARINEROS EN FUNCIÓN DE SUS GLUTENINAS DE ALTO PESO MOLECULAR
}

\author{
RHEOLOGICAL PROPERTIES STABILITY OF BREAD WHEATS THROUGH ENVIROMENTS \\ AS A FUNCTION OF THEIR HIGH MOLECULAR WEIGHT GLUTENINS
}

\author{
Micaela De la O Olán ${ }^{1}$, Eduardo Espitia Rangel*2, José D. Molina Galán , Héctor E. Villaseñor Mir², \\ Higinio López Sánchez ${ }^{3}$, Roberto J. Peña Bautista ${ }^{4}$ y Amalio Santacruz Varela ${ }^{1}$
}

\begin{abstract}
${ }^{1}$ Postgrado de Recursos Genéticos y Productividad, Colegio de Postgraduados, Campus Montecillo. Km. 36.5 Carr. México-Texcoco. 56230, Montecillo, Texcoco, Edo. de México. ${ }^{2}$ Programa de Trigo, Campo Experimental Valle de México, Instituto Nacional de Investigaciones Forestales, Agrícolas y Pecuarias. 56230, Chapingo, Edo. de México. ${ }^{3}$ Colegio de Postgraduados, Campus Puebla. Km. 125.5 Carretera Federal México-Puebla. 72720, Santiago, Momoxpan, Municipio de San Pedro Cholula, Puebla. ${ }^{4}$ Programa de Trigo, Centro Internacional de Mejoramiento de Maíz y Trigo. Apdo Postal 6-641. 06600, México, D. F.
\end{abstract}

* Autor para correspondencia (espitia.eduardo@inifap.gob.mx)

\section{RESUMEN}

Con el propósito de determinar las combinaciones alélicas de gluteninas de alto peso molecular (G-APM) y estudiar la estabilidad conferida por dichas combinaciones sobre las propiedades reológicas de la masa en trigos harineros mexicanos, se evaluaron las variedades 'Náhuatl F2000', 'Pavón F76', 'Temporalera M87', 'Rebeca F2000', 'Tlaxcala F2000', 'Gálvez M87', 'Zacatecas VT74', 'Romoga F96', 'Juchi F2000' y 'Batán F96'. Los genotipos se sembraron en 22 ambientes de temporal o secano en los Estados de México, Tlaxcala, Jalisco, Oaxaca, Puebla, Guanajuato, Morelos y Querétaro, durante los ciclos P-V/1999 y 2000, bajo un diseño experimental de bloques completos al azar con dos repeticiones. Se evaluaron las propiedades viscoelásticas (reológicas) de la masa mediante las variables tiempo de amasado $(\mathrm{min})$, alveograma-W, alveograma-P/L y alveograma-P/G. Se estimaron los parámetros de estabilidad mediante cuatro modelos diferentes. Las variedades 'Náhuatl F2000', 'Pavón F76' y 'Temporalera M87' poseen las subunidades de G-APM en Glu-A1 2*, Glu-B1 17+18 y Glu-D1 $5+10$ (combinación 1); las variedades 'Rebeca F2000', 'Tlaxcala F2000', 'Gálvez M87', 'Zacatecas VT74' y 'Romoga F96' en Glu-A1 1, Glu-B1 17+18 y Glu-D1 5+10 (combinación 2); y las variedades 'Juchi F2000' y 'Batán F96' en Glu-A1 2*, Glu-B1 7+9 y Glu-D1 $5+10$ (combinación 3). Hubo diferencias significativas $(P \leq 0.01)$ en todas las propiedades viscoelásticas para las combinaciones de GAPM. La combinación alélica 2 fue la más estable sobre las propiedades reológicas de la masa, y fue superior en algunas características viscoeláticas como fuerza de la masa. Por tanto, las variedades que poseen esta combinación son muy deseables para la industria mecanizada.

Palabras clave: Triticum aestivum, alveograma, calidad, interacción genotipo-ambiente, propiedades viscoelásticas.

\section{SUMMARY}

To determine the allelic combinations of high molecular weight glutenins (HMW-G) and the stability they confer over rheologic properties of the dough in Mexican bread wheats, ten varieties were evaluated. These included 'Nahuatl F2000', 'Pavón F76', 'Temporalera M87', 'Rebeca F2000', 'Tlaxcala F2000', 'Gálvez M87', 'Zacatecas VT74', 'Romoga F96', 'Juchi F2000' and 'Batán F96'. Genotypes were planted during the 1999 and 2000 SpringSummer cycle at 22 rainfed environments in the states of México, Tlaxcala, Jalisco, Oaxaca, Puebla, Guanajuato, Morelos and Querétaro. A completely randomized blocks design with 2 replications was used. Viscoelastic (rheologic) properties of the dough such as kneading time $(\mathrm{min}), \mathrm{W}$-alveogram, $\mathrm{P} / \mathrm{L}$ alveogram and P/G-alveogram were evaluated. Data were analyzed by estimating stability parameters through four different models. Varieties 'Náhuatl F2000', 'Pavón F76' and 'Temporalera M87' have HMW-G subunits as follows: $2^{*}$ at $G l u-A 12^{*}, 17+18$ at $G l u-B 1$ and $5+10$ at Glu-D1 (combination 1); varieties 'Rebeca F2000', 'Tlaxcala F2000', 'Gálvez M87', 'Zacatecas VT74' and 'Romoga F96' have 1 at $G l u-A 1,17+18$ at $G l u-B 1$ and y $5+10$ at $G l u-D 1$ (combination 2), and varieties Juchi F2000 and Batán F96 have 2* at Glu-A1, 7+9 at Glu-B1 and 5+10 at Glu-D1 (combination 3). Highly significant differences were found for all the evaluated viscoelastic properties for HMW-G combinations. The estimation of dough stability parameters showed that combination 2 was the most stable over rheologic properties; in addition, such a combination was superior in some viscoelatic characteristics as dough strength; therefore, varieties having such combination are highly desirable for the mechanized industry.

Index words: Triticum aestivum, alveogram, quality, genotypeenvironment interaction, viscoelastic properties.

\section{INTRODUCCIÓN}

El trigo (Triticum aestivum L.) es actualmente el cultivo de mayor superficie sembrada en el mundo (FAOSTAT, 2008). Las masas de harina de trigo poseen una red de proteínas de gluten que les confieren propiedades visco-elásticas (reológicas), de las cuales las de amasado, fuerza y extensibilidad son las más importantes. La medición directa de tales propiedades 
permite conocer y validar los efectos de las combinaciones de gluteninas y gliadinas, además de aportar información sobre los parámetros que requiere la industria, para determinar si la calidad de una variedad es adecuada para su proceso y producto a elaborar (Peña, 2002).

Una clase de proteínas del endospermo que conforman el gluten, las gluteninas, es la responsable más directa de la variación en calidad. La fracción de gluteninas de alto peso molecular (G-APM) se puede analizar por métodos bioquímicos no influenciados por el ambiente, ni por el contenido proteínico, y ha sido asociada con propiedades de la masa (Hoseney, 1991). Las subunidades de gluteninas de alto peso molecular son codificadas por genes en tres loci diferentes, Glu$A 1$, Glu-B1 y Glu-D1, ubicados en los cromosomas 1A, 1B y 1D respectivamente (Southan y MacRitchie, 1999). Entre las gluteninas con efecto conocido sobre las características de la fuerza del gluten se encuentran las subunidades $1,2 *, 7+8,7+9,13+16,17+18 \mathrm{y}$ $5+10$, con efecto positivo; las subunidades $7+9$ y $2+12$, con efecto intermedio; y las subunidades 0,7 , $20,13+19,3+12$ y $4+12$, con efecto negativo (Pogna et al., 1992). El efecto genético individual de los alelos de las G-APM sobre las propiedades reológicas ha sido el más estudiado, no así el producto de las combinaciones alélicas en las propiedades del gluten (Peña $e t$ al., 2002).

El común denominador de los programas de evaluación de genotipos es el comportamiento inestable de éstos en los ambientes de prueba. En ocasiones los resultados llegan a discrepar tanto de lo esperado, que resulta difícil tomar una decisión con respecto al mejor genotipo. La inconsistencia de resultados de tales evaluaciones puede tener su origen en el grado de diversidad de los genotipos y de los ambientes, o de ambos (Molina, 1992).

Lin et al. (1986) describieron tres tipos de estabilidad; el primero se refiere a una mínima varianza entre ambientes; el segundo define a un genotipo estable cuando su respuesta es paralela a la de la media de todos los genotipos ensayados, como propusieron Shukla (1972), y Finlay y Wilkinson (1963); y un tercero asociado al genotipo ideal cuyo cuadrado medio de desviaciones de regresión sea pequeño, donde el índice ambiental se considera como variable independiente (parámetro $\mathrm{Sd}_{\mathrm{i}}$ del método de Eberhart y Russell (1966); parámetro $\lambda_{i}$ Tai (1971). Lin et al. (1986) también definieron un cuarto concepto de estabilidad referido a variaciones pequeñas de un genotipo en una misma localidad pero en diferentes años. Es importante conocer el modelo que mejor se ajuste a un determinado conjunto de datos, para elegir la variedad más estable y con mejor expresión en determinados ambientes (Rodríguez et al., 2002).

Dada la necesidad de elaborar productos cada vez más diversos para satisfacer un mercado siempre ávido de mejoras y novedades, y a que la industria y la exportación demandan trigos y harinas de características definidas, se plantea que ante las diversas subregiones y la amplia disponibilidad de variedades de trigo que tiene México, satisfacer esos requerimientos resulta factible si se conoce la variación de la calidad a través de ambientes. Por ello, el objetivo de esta investigación fue determinar las combinaciones alélicas de G-APM y estudiar la estabilidad que confieren dichas combinaciones sobre las propiedades reológicas de la masa, encontradas en variedades de trigo harinero cultivadas en áreas de temporal o secano.

\section{MATERIALES Y MÉTODOS}

Se utilizaron 10 variedades de trigo harinero de temporal: 'Juchi F2000', 'Náhuatl F2000', 'Tlaxcala F2000', 'Rebeca F2000', 'Pavón F76', 'Gálvez M87', 'Temporalera M87', 'Batán F96', 'Romoga F96' y 'Zacatecas VT74'. Los genotipos se sembraron en 11 localidades (Juchitepec, Tecamac y Coatepec en el Estado de México; Nanacamilpa y Apizaco en el Estado de Tlaxcala; Jesús María en Jalisco; Cuyuaco en Puebla; Roque en Guanajuato; Buenavista en Morelos, Amealco en Querétaro; y Santiago Tillo en Oaxaca), durante dos ciclos agrícolas (primavera-verano de 1999 y 2000), lo que generó un total de 22 ambientes de temporal. Se utilizó un diseño experimental de bloques completos al azar con dos repeticiones. La unidad experimental consistió de cuatro surcos de $3 \mathrm{~m}$ separados a $30 \mathrm{~cm}$. Las siembras se realizaron de acuerdo con las recomendaciones del Instituto Nacional de Investigaciones Forestales, Agrícolas y Pecuarias (INIFAP) para cada región.

Los análisis para las variables de calidad se efectuaron en el Laboratorio de Calidad de Trigo del Centro Internacional de Mejoramiento de Maíz y Trigo (CIMMYT), mediante análisis reológicos de la masa e identificación de los patrones electroforéticos de las GAPM.

Para obtener harina refinada las muestras de grano se molieron en un molino Brabender Quadramat ${ }^{\circledR}$ (C.W. Brabender OHG, Germany). Las propiedades reológicas de la masa se determinaron mediante las variables: tiempo 
de amasado, alveograma-W, alveograma-P/L y alveograma-P/G. El tiempo de amasado en minutos, se determinó con el mixógrafo de Swanson ${ }^{\circledR}$ (National Manufacturing Co., Lincoln, NE, USA), que considera a la absorción de agua de acuerdo con el contenido de proteína [método 54-40A de la American Association of Cereal Chemists (AACC, 1995)]; aquí, un mayor tiempo de amasado corresponde a mayor fuerza de gluten y viceversa. Las variables alveográficas $\mathrm{W}, \mathrm{P} / \mathrm{L}$ y $\mathrm{P} / \mathrm{G}$ fueron obtenidas en el alveógrafo de Chopin ${ }^{\circledR}$ (Trippette y Renaud, París, Francia), con el método 54-30A de la AACC (1995); para ello se pesaron $50 \mathrm{~g}$ de harina refinada y se agregó una solución acuosa de $\mathrm{NaCl} 2.5 \%$, y la cantidad de agua se corrigió de acuerdo con la dureza del grano. El alveograma-W es una medida de la fuerza de la masa $\left(10^{-4} \mathrm{~J}\right)$, donde valores menores a 200 corresponden a un gluten débil, entre 200 y 300 a un gluten medio fuerte, y mayores a 300 a un gluten fuerte. El alveograma-P/L, que relaciona la altura y la longitud del alveograma, se registró mediante una escala adimensional de 0.1 a 6 ; la mayor extensibilidad corresponde a los valores menores. El alveograma-P/G, que es una relación entre la altura y el índice de expansión (tenacidad/extensibilidad), se midió con la escala adimensional de 1.5 a 14; la mayor extensibilidad corresponde a valores menores.

La identificación de G-APM se hizo por electroforesis en geles de poliacrilamida (SDS-PAGE) (Peña, 2002). Se colocaron $40 \mathrm{~g}$ de harina integral en un tubo eppendorf durante $20 \mathrm{~min}$ a temperatura ambiente $\left(20-22{ }^{\circ} \mathrm{C}\right)$, con $600 \mu \mathrm{L}$ de solución amortiguadora $\mathrm{HCl} 62.5 \mathrm{mM}(\mathrm{pH}$ 6.8) que contenía $12 \%(\mathrm{p} / \mathrm{v})$ de glicerol, $2 \%(\mathrm{p} / \mathrm{v})$ de dodecil sulfato sódico, $0.003 \%(\mathrm{p} / \mathrm{v})$ de azul de bromofenol y $5 \%$ de 2-mercaptoetanol. Después de la extracción, las muestras se centrifugaron por $5 \mathrm{~min}$ a 10 $000 \mathrm{rpm}$ (aproximadamente $8000 \mathrm{~g}$ ). Se utilizaron $4 \mu \mathrm{L}$ de extracto para la separación de proteínas sobre un gel de $18 \mathrm{~cm}$ de acrilamida a $10 \%$. El corrimiento electroforético se efectuó a $11 \mathrm{~mA}$ por $16.5 \mathrm{~h}$ a $15{ }^{\circ} \mathrm{C}$. Para revelar las bandas de proteína los geles permanecieron inmersos por $8 \mathrm{~h}$ en solución de tinción con solución acuosa a $0.13 \%$ de cumasina azul brillante R250, alcohol butílico y ácido acético (53:40:7, v/v). La destinción del gel se hizo con agua durante la noche. Las subunidades de alto peso molecular fueron identificadas de acuerdo con la nomenclatura de Payne et al. (1987), y como referencia se usó el patrón de bandeo de las variedades 'Chinese Spring', 'Opata', 'Pitic' y 'Pavón'.

Los datos fueron procesados para estimar parámetros de estabilidad mediante los modelos: modelo I, varianza de estabilidad de Shukla (1972); modelo II: regresión de Finlay y Wilkinson (1963); modelo III: parámetros de estabilidad de Eberhart y Russell (1966); y modelo IV: varianza ambiental (Lin et al., 1986), todos ellos descritos por Piepho (1999). Se utilizó el paquete SAS, versión 6.12 (SAS Institute, 1997), del cual se empleo el procedimiento MIXED con las medias de combinación de G-APM-ambiente, donde las combinaciones se tomaron como factor fijo y los ambientes como factor aleatorio. La comparación de medias ajustadas se hizo por cuadrados mínimos mediante la opción PDIFF, cuya diferencia estadística fue determinada por la prueba de $t$ de Student.

Las pruebas de bondad de ajuste de los datos a los modelos propuestos se hicieron mediante máxima verosimilitud restringida (REML), al usar la proporción estadística entre el valor del parámetro de máxima verosimilitud restringida del modelo general (LLR), en este caso el modelo de la varianza ambiental y el valor respectivo del modelo bajo consideración. El LLR se distribuye como una $\chi^{2}$ con $g l$ grados de libertad proveniente de la diferencia de parámetros $(p)$ en la estructura varianza-covarianza del modelo de la varianza ambiental y el modelo reducido en cuestión. Dicha $\chi^{2}$ se calculó mediante la fórmula propuesta por Oman (1991) y Wolfinger (1993), donde: $\chi^{2}=-2$ (LLR del modelo bajo consideración - LLR del modelo de la varianza ambiental). Una segunda estrategia para seleccionar el modelo más adecuado fue el criterio de información de Akaike (AIC), citado por Wolfinger (1993), el cual se calcula mediante la ecuación: $\mathrm{AIC}=\mathrm{LLR}-p$, donde $p$ es el número de parámetros en la estructura varianzacovarianza de cada modelo. A mayor valor de AIC, el modelo se considera más adecuado.

\section{RESULTADOS Y DISCUSIÓN}

En la Figura 1 se muestra que las variedades 'Náhuatl F2000', 'Pavón F76' y 'Temporalera M87' poseen las subunidades de G-APM en Glu-A1 2*, en Glu-B1 17+18 y en Glu-D1 5+10, por lo que se agruparon para conformar la Combinación 1; las variedades 'Rebeca F2000', 'Tlaxcala F2000', 'Gálvez M87', 'Zacatecas VT74' y 'Romoga F96' poseen las subunidades de GAPM en Glu-Al 1, en Glu-B1 17+18 y en Glu-D1 5+10, integrando la Combinación 2; las variedades 'Juchi F2000' y 'Batán F96' poseen las subunidades de G-APM en Glu-A1 2*, en Glu-B1 7+9 y en Glu-D1 5+10, agrupadas en la Combinación 3. 


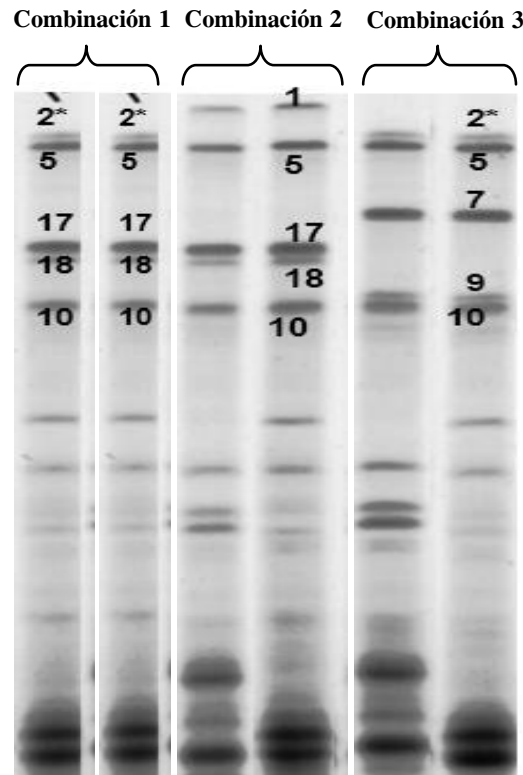

Figura 1. Gel de poliacrilamida en poblaciones de trigos harineros mexicanos, que muestra alelos de G-APM.

Con los cuatro modelos de estabilidad se encontraron diferencias significativas $(\mathrm{P} \leq 0.01)$ en todas las variables reológicas para las combinaciones de G-APM; es decir, las propiedades viscoelásticas de la masa conferidas por las combinaciones de gluteninas difirieron entre ambientes de prueba. En el Cuadro 1 se muestran los valores de bondad de ajuste $\left(\mathrm{R}^{2}\right)$ de los diferentes modelos probados. El mayor valor de AIC para alveograma-W y alveogramaP/G correspondió al modelo de varianza de estabilidad, por lo que de acuerdo con Wolfinger (1993), la estabilidad de las combinaciones de G-APM a través de ambientes deberá estimarse con este modelo; en contraste, en alveograma-P/L y tiempo de amasado el modelo que mejor se ajustó fue el de regresión de Finlay y Wilkinson (1963). De manera general, cada propiedad reológica se ajustó a modelos diferentes, igual a lo observado por Robert y Denis (1996), quienes con 10 variedades de trigo en 14 ambientes encontraron interacción significativa de la calidad $\mathrm{x}$ ambiente, lo que indicó que las variables reológicas deben ser analizadas por separado para definir el método que mejor se ajuste en la evaluación de la estabilidad de la calidad panadera.

Los parámetros relevantes de REML con errores estándar (ee) para la estructura varianza-covarianza de los diferentes modelos de estabilidad evaluados (Cuadro 2), muestran que la combinación de G-APM 1, 17+18, $5+10$ fue la más estable, de acuerdo con los modelos aplicados en todas las propiedades reológicas de la masa, ya que en esta combinación hubo el menor valor de $\sigma_{i i}$, el menor valor de varianza de la estabilidad $\sigma_{(i)}^{2}$, el menor valor absoluto del coeficiente de regresión $\left(\lambda_{i}\right)$, de acuerdo con los análisis de Finlay-Wilkinson (1963) y Eberhart-Russell (1966) y la menor varianza de las desviaciones $\left(\sigma_{(i i)}^{2},\right)$, de acuerdo con el modelo de EberhartRussell. En este sentido las variedades más estables en calidad panadera fueron 'Rebeca F2000', 'Tlaxcala F2000', 'Gálvez M87', 'Zacatecas VT74' y 'Romoga F96', en virtud de que poseen dicha combinación.

Cuadro 1. Pruebas de bondad de ajuste en variables reológicas de tres combinaciones de G-APM en trigos harineros de temporal en 22 ambientes de evaluación. P-V, 1999 y 2000.

\begin{tabular}{|c|c|c|c|c|c|c|c|}
\hline Variable & Modelo & $p$ & AIC & $-2 L L R$ & $\chi^{2}$ & $\mathrm{gl}$ & $\mathrm{P}$ \\
\hline \multirow[t]{4}{*}{ Alveograma-W } & Modelo I & 4 & -345.9 & 683.8 & 75.4 & 14 & $<0.0001$ \\
\hline & Modelo II & 4 & -346.6 & 685.2 & 74.0 & 14 & $<0.0001$ \\
\hline & Modelo III & 6 & -347.5 & 682.7 & 76.5 & 12 & $<0.0001$ \\
\hline & Modelo IV & 18 & -397.6 & 759.2 & & & \\
\hline \multirow[t]{4}{*}{ Alveograma $\mathrm{P} / \mathrm{G}$} & Modelo I & 4 & -60.7 & 113.4 & 55.1 & 14 & $<0.0001$ \\
\hline & Modelo II & 4 & -62.0 & 116.1 & 52.4 & 14 & $<0.0001$ \\
\hline & Modelo III & 6 & -60.9 & 109.9 & 58.6 & 12 & $<0.0001$ \\
\hline & Modelo IV & 18 & -102.2 & 168.5 & & & \\
\hline \multirow[t]{3}{*}{ Alveograma $\mathrm{P} / \mathrm{L}$} & Modelo I & 4 & 10.6 & -29.2 & 0.95 & 14 & $<0.0001$ \\
\hline & Modelo II & 6 & 13.3 & -38.6 & 5.65 & 12 & $<0.0001$ \\
\hline & Modelo III & 18 & 31.6 & 27.3 & & & \\
\hline \multirow[t]{4}{*}{ Tiempo de Amasado } & Modelo I & 4 & -50.3 & 92.6 & 28.1 & 14 & $<0.0001$ \\
\hline & Modelo II & 4 & -46.6 & 85.3 & 32.55 & 14 & $<0.0001$ \\
\hline & Modelo III & 6 & -48.0 & 84.1 & 33.15 & 12 & $<0.0001$ \\
\hline & Modelo IV & 18 & -93.2 & 150.4 & & & \\
\hline
\end{tabular}

Modelo I: varianza de estabilidad (Shukla, 1972); Modelo II: regresión (Finlay y Wilkinson, 1963); Modelo III: regresión (Eberhart-Rusell, 1966); Modelo IV: varianza ambiental (Lin et al., 1986). p: número de parámetros en la estructura varianza-covarianza; AIC: criterio de información de Akaike; LLR: parámetro de máxima verosimilitud restringida; gl: grados de libertad = $p$ (modelo de la varianza ambiental) $p$ (modelo bajo consideración). 
Cuadro 2. Parámetros estimados de REML (máxima verosimilitud restringida) con errores estándar (ee) para la estructura varianza-covarianza de los modelos de estabilidad aplicados a las variables reológicas de 3 combinaciones de G-APM de variedades de trigos harineros mexicanos en 22 ambientes. P-V, 1999 y 2000.

\begin{tabular}{llrrr}
\hline Variable & \multicolumn{1}{c}{ Modelo } & Combinación 1 & Combinación 2 & Combinación 3 \\
\hline Alveograma-W & Modelo I- $\sigma_{(i)}^{2}(\mathrm{ee})$ & $1756.7(630.0)$ & $535.0(366.4)$ & $602.0(378.0)$ \\
& Modelo II- $\lambda_{i}(\mathrm{ee})$ & $98.0(16.5)$ & $83.3(14.4)$ & $83.9(14.5)$ \\
& Modelo IIIa- $\lambda_{i}(\mathrm{ee})$ & $1572.2(610)$ & $561.0(339)$ & $611.8(349)$ \\
& Modelo IIIb- $\sigma_{(i i)}^{2}(\mathrm{ee})$ & $95.3(17.1)$ & $84.6(14.1)$ & $85.0(14.2)$ \\
Alveograma P/G & Modelo IV- $\sigma_{(i i)}(\mathrm{ee})$ & $10672.0(3293)$ & $7734.6(2386)$ & $7847.1(2421)$ \\
& Modelo I- $\sigma_{(i)}^{2}(\mathrm{ee})$ & $0.149(0.05)$ & $0.042(0.03)$ & $0.181(0.06)$ \\
& Modelo II- $\lambda_{i}(\mathrm{ee})$ & $0.759(0.14)$ & $0.829(0.15)$ & $0.740(0.14)$ \\
& Modelo IIIa- $\lambda_{i}(\mathrm{ee})$ & $0.165(0.05)$ & $0.000(-)$ & $0.188(0.05)$ \\
Alveograma P/L & Modelo IIIb- $\sigma_{(i i)}^{2}(\mathrm{ee})$ & $0.745(0.14)$ & $0.881(0.13)$ & $0.717(0.14)$ \\
& Modelo IV- $\sigma_{(i i)}(\mathrm{ee})$ & $0.720(0.22)$ & $0.776(0.23)$ & $0.704(0.21)$ \\
& Modelo II- $\lambda_{i}(\mathrm{ee})$ & $0.222(0.042)$ & $0.259(0.047)$ & $0.292(0.052)$ \\
& Modelo IIIa- $\lambda_{i}(\mathrm{ee})$ & $0.011(0.003)$ & $0.000(-)$ & $0.025(0.007)$ \\
& Modelo IIIb- $\sigma_{(i i)}^{2}(\mathrm{ee})$ & $0.226(0.041)$ & $0.275(0.042)$ & $0.275(0.054)$ \\
& Modelo IV- $\sigma_{(i i)}(\mathrm{ee})$ & $0.062(0.019)$ & $0.076(0.023)$ & $0.101(0.031)$ \\
& Modelo I- $\sigma_{(i)}^{2}(\mathrm{ee})$ & $0.108(0.057)$ & $0.132(0.057)$ & $0.040(0.041)$ \\
& Modelo II- $\left.\lambda_{i}\right) \lambda_{i}(\mathrm{ee})$ & $0.691(0.121)$ & $0.835(0.141)$ & $0.575(0.106)$ \\
& Modelo IIIa- $\lambda_{i}(\mathrm{ee})$ & $0.100(0.040)$ & $0.051(0.042)$ & $0.054(0.025)$ \\
& Modelo IIIb- $\sigma_{(i i)}^{2}(\mathrm{ee})$ & $0.677(0.126)$ & $0.844(0.141)$ & $0.580(0.104)$ \\
& Modelo IV- $\sigma_{(i i)}(\mathrm{ee})$ & $0.558(0.172)$ & $0.764(0.235)$ & $0.390(0.120)$ \\
\hline
\end{tabular}

Modelo I: varianza de estabilidad (Shukla, 1972) $\sigma_{(i)}^{2}$; Modelo II: regresión (Finlay y Wilkinson, 1963) $\lambda_{i}$; Modelo IIIa: regresión (Eberhart-Rusell, 1966) $\lambda_{i}$; Modelo IIIb: regresión (Eberhart-Rusell, 1966) $\sigma_{(i i)}^{2}$; Modelo IV: varianza ambiental (Lin et al., 1986) $\sigma_{(i i)}$. Combinación 1: 2*, 17+18, $5+10$; Combinación 2: 1, 17+18, 5+10; Combinación 3: 2*, 7+9, 5+10.

Las medias y los errores estándar fueron consistentes a través de todos los modelos. La combinación menos estable fue la $2^{*}, 17+18,5+10$. Los errores estándar de los parámetros estimados fueron altos en todas las combinaciones para la variable alveograma-W, lo que podría indicar que 22 ambientes no es una muestra adecuada para estimar de manera confiable la estabilidad (Piepho, 1999), o bien que el área de ambientes involucrados, que incluyen tipos de suelo distintos y variables climáticas diversas, es bastante contrastante (Molina, 1992). Sin embargo, la combinación alélica menos estable para alveograma-W, resultó con estabilidad para alveograma-P/G, alveograma $\mathrm{P} / \mathrm{L}$ y tiempo de amasado, cuyos errores estándar fueron relativamente bajos, lo que sugiere una tendencia de menor interacción con el ambiente (Piepho, 1999).

Las medias de las cuatro variables reológicas con sus respectivos errores estándar para los diferentes modelos estudiados se muestran en el Cuadro 3, donde se aprecia que las medias fueron iguales y los errores estándar muy similares a través de todos los modelos. Además de estable, se desea que una combinación de G-APM tenga una media de alveograma-W alta, una extensibilidad alta (menores valores de alveogramas $\mathrm{P} / \mathrm{G}$ y $\mathrm{P} / \mathrm{L}$ ) y mayor tiempo de amasado para la industria mecanizada. De acuerdo con el modelo de la varianza de estabilidad, la combinación $1,17+18,5+10$ obtuvo el mayor valor para la fuerza de la masa (alveograma-W); es decir, además de ser la combinación más estable, es la que mayor fuerza posee y produce la combinación más deseable. En alveograma-P/G el menor valor lo obtuvo la combinación $2^{*}, 7+9,5+10$, que indica que las variedades que poseen dicha combinación tienen buena extensibilidad de la masa. Con el modelo de regresión de Finlay y Wilkinson (1963) sobre la variable alveograma $\mathrm{P} / \mathrm{L}$, el menor valor correspondió a la combinación $2 *, 7+9,5+10$, que corresponde a una buena extensibilidad de la masa. Para tiempo de amasado el mayor valor correspondió con mayor fuerza de la masa, y la mejor combinación resultó ser $1,17+18,5+10$. Estos resultados indican las combinaciones alélicas más deseables en la industria harinera y sobre todo cuál posee las propiedades reológicas más estables (Peña et al., 2002).

En el Cuadro 4 se presentan las comparaciones de medias generadas por el modelo de varianza de la estabilidad para alveograma-W y alveograma-P/G, y por el modelo de Finlay y Wilkinson (1963) para alveograma$\mathrm{P} / \mathrm{L}$ y tiempo de amasado. La mejor combinación para fuerza de la masa, que involucra las variables alveograma-W y tiempo de amasado, fue la segunda que incluye los alelos de G-APM $1,17+18,5+10$; por tanto, las variedades que poseen dicha combinación ('Rebeca 
F2000', 'Tlaxcala F2000', 'Gálvez M87', 'Zacatecas VT74' y 'Romoga F96') son las más deseables. Para extensibilidad de la masa, que incluye las variables alveograma-P/G y alveograma-P/L, la mejor combinación resultó ser también $1,17+18,5+10$, mientras que la combinación menos estable fue la tercera, con los alelos
$2 *, 7+9, \quad 5+10$. Lo anterior indica que existen variedades relativamente recientes (de la serie 2000) con buena calidad panadera, y algunas de ellas poseen estabilidad y propiedades reológicas adecuadas para la industria de panificación mecanizada.

Cuadro 3. Medias de variables reológicas con errores estándar (ee) obtenidas por diferentes modelos de estabilidad para tres combinaciones de G-APM en variedades de trigo harinero de temporal cultivadas en 22 ambientes. P-V, 1999 y 2000.

\begin{tabular}{llccc}
\hline Variable & Modelo & Combinación 1 & Combinación 2 & Combinación 3 \\
\hline Alveograma-W & Modelo I & $413.18(20.4)$ & $444.41(19.0)$ & $362.64(19.1)$ \\
\multicolumn{1}{c}{$\left(10^{-4} \mathrm{~J}\right)$} & Modelo II & $413.18(21.8)$ & $444.41(18.8)$ & $362.64(18.9)$ \\
& Modelo III & $413.18(22.0)$ & $444.41(18.7)$ & $362.64(18.8)$ \\
Alveograma P/G & Modelo IV & $413.18(22.0)$ & $444.41(18.7)$ & $362.64(18.7)$ \\
(Escala 1.5-14) & Modelo I & $4.10(0.193)$ & $4.44(0.180)$ & $3.81(0.196)$ \\
& Modelo II & $4.10(0.179)$ & $4.44(0.192)$ & $3.81(0.175)$ \\
Alveograma P/L & Modelo III & $4.10(0.181)$ & $4.44(0.187)$ & $3.81(0.178)$ \\
(Escala 0-6) & Modelo IV & $4.10(0.181)$ & $4.44(0.187)$ & $3.81(0.178)$ \\
Tiempo de & Modelo II & $0.834(0.052)$ & $0.921(0.060)$ & $0.816(0.066)$ \\
Amasado (min) & Modelo III & $0.834(0.053)$ & $0.921(0.058)$ & $0.816(0.067)$ \\
& Modelo IV & $0.834(0.053)$ & $0.921(0.058)$ & $0.816(0.067)$ \\
& Modelo I & $3.068(0.155)$ & $3.576(0.158)$ & $2.953(0.145)$ \\
& Modelo II & $3.068(0.158)$ & $3.576(0.187)$ & $2.953(0.134)$ \\
\hline
\end{tabular}

Modelo I: varianza de estabilidad (Shukla, 1972); Modelo II: regresión (Finlay y Wilkinson, 1963); Modelo III: regresión (Eberhart-Rusell, 1966); Modelo IV: varianza ambiental (Lin et al., 1986). Combinación 1: 2*, 17+18, 5 + 10; Combinación 2: $1,17+18,5+10 ;$ Combinación 3: 2*, 7+9, 5+10.

Cuadro 4. Comparación de medias para variables reológicas de 3 combinaciones de G-APM en variedades de trigo harinero de temporal, en 22 ambientes de evaluación. P-V, 1999 y 2000.

\begin{tabular}{lcccccc}
\hline Variable & Combinación & Diferencia & Error & gl & Valor de t & Pr $>\mathrm{t}$ \\
\hline Alveograma-W (10-4 J) & 1 vs. 2 & -31.220 & 28.920 & 41 & -1.08 & 0.28 \\
& 1 vs. 3 & 50.540 & 29.010 & 41 & 1.74 & 0.08 \\
Alveograma P/G (Escala 1.5-14) & 2 vs. 3 & 81.770 & 26.610 & 42 & 3.07 & 0.003 \\
& 1 vs. 2 & -0.334 & 0.260 & 41.9 & -1.28 & 0.208 \\
Alveograma P/L (Escala 0-6) & 1 vs. 3 & 0.292 & 0.255 & 42 & 1.15 & 0.257 \\
& 2 vs. 3 & 0.626 & 0.259 & 41.9 & 2.41 & 0.020 \\
Tiempo de amasado (min) & 1 vs. 2 & -0.087 & 0.079 & 41.6 & -1.10 & 0.276 \\
& 1 vs. 3 & 0.018 & 0.086 & 39.7 & 0.21 & 0.834 \\
& 2 vs. 3 & 0.106 & 0.090 & 41.1 & 1.18 & 0.246 \\
\hline
\end{tabular}

Combinación 1: 2*, 17+18, $5+10$; Combinación 2: 1, 17+18, 5+10; Combinación 3: 2*, 7+9, 5+10. 


\section{CONCLUSIONES}

Las variedades 'Náhuatl F2000', 'Pavón F76' y 'Temporalera M87' poseen las subunidades de G-APM en Glu-A1 2*, Glu-B1 17+18 y Glu-D1 5+10, por lo que se agruparon para conformar la Combinación 1; las variedades 'Rebeca F2000', 'Tlaxcala F2000', 'Gálvez M87', 'Zacatecas VT74' y 'Romoga F96' poseen las subunidades de G-APM en Glu-A1 1, Glu-B1 17+18 y Glu-D1 5+10, e integrando la Combinación 2; mientras que las variedades 'Juchi F2000' y 'Batán F96' poseen las subunidades de G-APM en Glu-A1 2*, Glu-B1 7+9 y Glu-D1 5+10, y se agrupan en la Combinación 3. La combinación de gluteninas de alto peso molecular con mayor estabilidad y con aceptable fuerza de la masa para la industria mecanizada fue $1,17+18,5+10$. La Combinación $2 *, 7+9,5+10$ proporciona buena extensibilidad de la masa, pero es la menos estable a través de ambientes.

\section{AGRADECIMIENTOS}

Al CONACYT (Proyecto 067698) a al INIFAP (Proyecto 2056029A) por el financiamiento otorgado para la realización de la presente investigación.

\section{BIBLIOGRAFÍA}

AACC (1995) Approved methods of the AACC. 9th ed. American Association of Cereal Chemists. St. Paul, MN, USA. 1268 p.

Eberhart S A, W A Rusell (1966) Stability parameters for comparing varieties. Crop Sci. 6:36-40.

FAOSTAT (2008) FAO Statistical Databases. Food and Agriculture Organization of the United Nations. Disponible en: http://faostat.fao.org/ (October 2008).

Finlay K W, A A Wilkinson (1963) The analysis of adaptation in a plant-breeding programme. Aus. J. Agric. Res. 14:742-754.

Hoseney R C (1991) Principios de Ciencia y Tecnología de Cereales. Ed. Acribia. Zaragoza, España. 334 p.
Lin C S, M R Binns, L P Lefkovitch (1986) Stability analysis: where do we stand. Crop Sci. 26:894-900.

Molina G JD (1992) Introducción a la Genética de Poblaciones y Cuantitativa (Algunas Implicaciones en Genotecnia). AGT Editor. México, D.F. 370 p.

Oman S D (1991) Multiplicative effects in mixed model analysis of variance. Biometrica 78: 729-739.

Payne P I, J A Seekings, A J Worland, M G Jarvis, L M Holt (1987) Allelic variations of glutenin subunits and gliadins and its effect on breadmaking quality in wheat: analysis of $\mathrm{F}_{5}$ progeny from Chinese Spring $\times$ Chinese Spring (Hope 1A) J. Cereal Sci. 6:103-118.

Peña R J (2002) Wheat for bread and other foods. In: Bread Wheat Improvement and Production. B C Curtis, S S Rajaram, H Gómez-MacPherson (eds). Food and Agriculture Organization of the United Nations. Rome. pp:483-542.

Peña R J, R Trethowan, W H Pfeiffer, M Van-Ginkel (2002) Quality (end-use) improvement in wheat: compositional, genetic, and environmental factors. In: A S Basra, LS Quality Improvement in Field Crops. Randhawa (eds). Food Products Press, impreso de Haworth Press, New York. pp:1-37.

Piepho H P (1999) Stability analysis using the SAS System. Agron. J. 91:160-165.

Pogna N E, R Rdaelli, T Dackevitch, A Curioni, A Dal Belin Perufo (1992) Benefits from genetics and molecular biology to improve the end use properties of cereals. In: Cereal Chemistry and Technology: a Long Past and a Bright Future. P. Feillet (ed). INRA. Montpellier, France. pp:83-93.

Robert N, J B Denis (1996) Stability of baking quality in bread wheat using several statistical parameters. Theor. Appl. Genet. 93:172-178.

Rodríguez P J E, J Sahagún C, H E Villaseñor M, J D Molina G, A Martínez G (2002) Estabilidad de siete variedades comerciales de trigo (Triticum aestivum L.). Rev. Fitotec. Mex. 25:143-151.

SAS Institute (1997) SAS/STAT Software: Changes and Enhancements through Release 6.12. SAS Institute. Cary, N.C. 5123 p.

Shukla G K (1972) Some statistical aspects of partitioning genotypeenviromental components of variability. Heredity 29:237-245.

Southan M, F MacRitchie (1999) Molecular weight distribution of wheat proteins. Cereal Chem. 76:827-836.

Wolfinger R D (1993) Covariance structure selection in general mixed models. Commun. Stat. Simul. Comp. 22:1079-1106. 